\title{
Genetic assessment of inbred chicken lines indicates genomic signatures of resistance to Marek's disease
}

\author{
Lingyang $\mathrm{Xu}^{1,2,3^{*}}$, Yanghua He ${ }^{1 \dagger}$, Yi Ding ${ }^{1}$, George E. Liư ${ }^{2}$, Huanmin Zhang ${ }^{4}$, Hans H. Cheng ${ }^{4}$,
} Robert L. Taylor Jr. ${ }^{5}$ and Jiuzhou Song ${ }^{*^{*}}$

\begin{abstract}
Background: Marek's disease (MD) is a highly contagious pathogenic and oncogenic disease primarily affecting chickens. However, the mechanisms of genetic resistance for MD are complex and not fully understood. MD-resistant line $\sigma_{3}$ and MD-susceptible line $7_{2}$ are two highly inbred progenitor lines of White Leghorn. Recombinant Congenic Strains (RCS) were developed from these two lines, which show varied susceptibility to MD.

Results: We investigated genetic structure and genomic signatures across the genome, including the line $\sigma_{3}$ and line $7_{2}$, six RCSs, and two reciprocally crossed flocks between the lines $\sigma_{3}$ and $7_{2}\left(F 1 \sigma_{3} \times 7_{2}\right.$ and $\left.F 17_{2} \times \sigma_{3}\right)$ using Affymetrix ${ }^{\oplus}$ Axiom ${ }^{\oplus}$ HD $600 \mathrm{~K}$ genotyping array. We observed 18 chickens from RCS lines were specifically clustered into resistance sub-groups distributed around line $6_{3}$. Additionally, homozygosity analysis was employed to explore potential genetic components related to $\mathrm{MD}$ resistance, while runs of homozygosity $(\mathrm{ROH})$ are regions of the genome where the identical haplotypes are inherited from each parent. We found several genes including SIK, SOX1, LIG4, SIK1 and TNFSF13B were contained in $\mathrm{ROH}$ region identified in resistant group (line $\sigma_{3}$ and RCS), and these genes have been reported that are contribute to immunology and survival. Based on $F_{S T}$ based population differential analysis, we also identified important genes related to cell death and anti-apoptosis, including AKT1, API5, CDH13, CFDP and USP15, which could be involved in divergent selection during inbreeding process.

Conclusions: Our findings offer valuable insights for understanding the genetic mechanism of resistance to MD and the identified genes could be considered as candidate biomarkers in further evaluation.
\end{abstract}

Keywords: Genetic structure, Genomic signature, Homozygosity, Marek's disease, Recombinant Congenic Strains (RCS)

\section{Background}

Poultry products are main components of our daily life. The complexity of food safety and quality issues facing the poultry industry are rapidly escalating, and many other critical issues (i.e., bioterrorism, environmental stewardship and global competitiveness) are emerging simultaneously. Marek's disease (MD) is a T cell lymphoma induced by the widespread and readily transmissible Marek's disease virus (MDV) [1]. MD is one of the main chronic infectious diseases threatening the poultry

\footnotetext{
* Correspondence: xulingyang@caas.cn; songj88@umd.edu

${ }^{\dagger}$ Lingyang Xu and Yanghua He contributed equally to this work.

${ }^{1}$ Department of Animal \& Avian Sciences, University of Maryland, College

Park, MD 20742, USA

Full list of author information is available at the end of the article
}

worldwide, and interest for its economic importance to the commercial poultry industry.

MDV transforms mainly CD4+ T cells and causes various clinical syndromes in chicken tissues, which include peripheral nerves, gonad, iris, muscle, viscera, and skin [2]. MDV is shed and transmitted between birds via epithelial cells of the feather follicle, dander, chicken house dust, feces and saliva. Vaccines were developed to control the disease but are not sterilizing allowing the virus to replicate and spread. Thus, in the last few decades, field strains of the virus have evolved resulting in new subtypes that the vaccines may not completely control [3]. MDV has proven to be a valuable comparative biomedical model organism for understanding the principles of human disease [4] and MD is a natural model for

(c) The Author(s). 2018 Open Access This article is distributed under the terms of the Creative Commons Attribution 4.0 International License (http://creativecommons.org/licenses/by/4.0/), which permits unrestricted use, distribution, and reproduction in any medium, provided you give appropriate credit to the original author(s) and the source, provide a link to the Creative Commons license, and indicate if changes were made. The Creative Commons Public Domain Dedication waiver (http://creativecommons.org/publicdomain/zero/1.0/) applies to the data made available in this article, unless otherwise stated. 
lymphomas overexpressing Hodgkin's disease antigen [5]. However, there is a shortage of genetic information about the virus host and etiological process leading to MDV uptake, dissemination, latency and tumor formation.

Currently, control strategies for MD predominantly rely on vaccination of chickens. But the vaccination cannot provide complete protection because of the changing nature of the disease itself and evolution of MDV with escalated virulence [4]. Recently, studies using integrating genomic approaches identified genes and molecular markers associated with MD disease resistance [6]. Genetic makers, quantitative trait loci (QTL) and genomics regions in a large reciprocal backcross (BC) were discovered between two partially inbred commercial White Leghorn layer chicken [7] and in an F6 advanced intercross population of commercial layer chickens [8]. Additionally, gene expression using transcript array $[9,10]$ and allele-specific expression analysis using RNA sequencing [11] have been utilized to understand response to MDV infection and MD genetic resistance.

To determine the genetic components pertaining to disease resistance, the Avian Disease and Oncology Laboratory (ADOL) has successfully developed inbred chicken lines resistant or susceptible to MD as a powerful experimental model for investigation of host resistance to this disease, the inbred lines $6_{3}$ and $7_{2}$. The percentage of chickens developing a tumor or tumors induced by a partially attenuated $\mathrm{vv}+$ strain of MDV differed significantly between the two lines of chickens. Genetic resistance to MD in chickens is commonly evaluated with MD incidence post-MDV challenge, which had been descripted in previous study [12]. Only $0-3 \%$ of line $6_{3}$ chickens developed tumors 8 wk post infection compared to $99 \%-100 \%$ of line $7_{2}$ chickens. Importantly, 19 recombinant congenic strains (RCS) have been developed using the inbred lines $6_{3}$ and $7_{2}$ as progenitor lines. The tumor incidence differs among RCS either vaccinated or unvaccinated. Thus, the varied resistance among the RCS can provide an ideal model to investigate genomic components that play vital roles in genetic resistance to MD. Although a previous study has demonstrated some genetic variability among the RCSs by microsatellite fingerprinting [2], genetic variations underlying varied susceptibility to MD in the RCS lines remains poorly understood.

In this study, we hypothesized that differential genomic signatures contribute to MD resistance in the RCS. Using ten lines, including line $6_{3}$ and line $7_{2}$, two hybrid F1 (F1 $6_{3}-7_{2}$ and F1 $7_{2}-6_{3}$ ) and six RCSs representing various capacity in resistance/susceptibility to $\mathrm{MD}$, we investigated genetic structure in the chicken lines based on Affymetrix ${ }^{\circ}$ Axiom $^{\circ}$ HD $600 \mathrm{~K}$ SNP array. Additionally, homozygosity analysis and genomic signatures were carried out to explore potential genetic mechanism of MD disease. Our studies revealed several genes related to regulation of cell death and anti-apoptosis, which are probably related to the resistance to MD.

\section{Methods}

\section{Experimental population}

In total, 30 chickens without treatments were selected and genotyped in this study. Among them, three were from each of the line $7_{2}$, line $6_{3}$, reciprocal cross $\mathrm{F} 1$ hybrid $7_{2} \times 6_{3}$, F1 hybrid $6_{3} \times 7_{2}$, and six recombinant congenic strains (RCSs) [C, J, M, N, S and X] [11]. RCSs were developed using line $6_{3}$ as the parental strain mated to line $7_{2}$ and then backcrossed to line $6_{3}$ twice followed by full-sib matings for about 20 generations. Eventually, diverse RCSs were generated and they contain $87.5 \%$ of line $6_{3}$ and $12.5 \%$ of line $7_{2}$ in the genetic background but with different MD resistance (Additional file 1: Figure S1) [3, 13]. In this study, six RCSs above were chosen to check their genetic background and reveal their particular resistant signatures.

\section{Genotyping and quality control}

Genomic DNA from red blood cells was extracted using the DNeasy Blood \& Tissue Mini Kit (QIAGEN). Genotyping for the 30 chickens was performed using the Affymetrix ${ }^{\circ}$ Axiom $^{\circ}$ Genome-Wide Chicken Genotyping Array $(600 \mathrm{~K})$ by the Affymetrix service facility according to the manufacturer's protocols [14]. Affymetrix adopts a preliminary call rate of $90 \%$ that is used by default to pass arrays for further data analysis. Successfully passed arrays were clustered and final genotypes were generated using the Axiom GT1 algorithm. Quality control was assessed in Genotyping Console v4.1.3. All chosen samples genotyped on Affymetrix SNP array met the $99 \%$ call rate.

\section{Multidimensional scaling and admixture analysis}

Quality control were considered using the following selection criteria: $\mathrm{MAF} \geq 0.01$ (including SNPs with $\mathrm{MAF} \geq 0.01$ ), geno $\geq 0.1$ (including only SNPs with a $90 \%$ genotyping rate or higher), and $P$ value of $x^{2}$ test for Hardy-Weinberg equilibrium $\geq 10^{-6}$. To avoid linkage distortion for the population structure analysis, SNPs were pruned for linkage disequilibrium (LD) using pair-wise genotype correction $\left(r^{2}>0.2\right)$ in $50 \mathrm{SNP}$ sliding widows with a step of 10 SNPs across the genome. To look for evidence of population substructure, we carried out Multidimensional Scaling (MDS) analysis using 5,064 LD filtered SNPs among all 30 chickens. Two dimensions in MDS analysis were calculated based on the identity-by-descent (IBD) pairwise distance among all chickens using PLINK, then MDS was plotted using the first-dimension values against the 
second-dimension values. To estimate the genetics admixture within 10 lines, STRUCTURE v2.3 was employed using the same LD filtered data set with 5,000 replicates and 2,000 burn-in cycles under the admixture model and correlated allele frequencies $[15,16]$.

\section{Phylogenetic analysis}

Neighbor-joining (NJ) tree was constructed based on the estimation of IBD. As line $7_{2}$ shows significantly susceptible to MD compared to the RCS, we used line $7_{2}$ as the outgroup for NJ trees analysis. Pairwise genetic distance (D) between chickens was calculated using PLINK, where $\mathrm{D}=1-[\mathrm{IBS} 2+0.5 \mathrm{IBS} 1) / \mathrm{N}]:$ IBS2 and IBS1 are the number of loci that share either 2 or 1 alleles identical by state (IBS), respectively, and the $\mathrm{N}$ is the number of loci [17, 18]. Then, phylogenetic tree was generated using FigTree 1.3.1 (http://tree.bio.ed.ac.uk/software/figtree/). In addition, Reynolds' distance between pairwise inbred lines was estimated using Arlequin version 3.5 [19].

\section{Differential genomic signature}

To investigate genome-wide patterns of genetic polymorphism related to susceptibility and resistance during the inbreeding process, the global $F_{\mathrm{ST}}$ was calculated for each single locus using Genepop software across all lines [20], which measured genetic differentiation between subpopulations using the approach previously described by Weir et al. [21]. Genomic signatures, implying divergent selection among inbred lines, can be recognized when $F_{\mathrm{ST}}$ values for the adjacent SNPs in a special window size were estimated to exceed the significant threshold [22]. We estimated average $F_{\mathrm{ST}}$ values of each nonoverlap region with 50 SNPs windows size. The top $1 \%$ regions were considered as candidate regions. Furthermore, we performed gene annotation based on genome assembly (Gallus_gallus-4.0) to identify the candidate genes involved in divergent selection during the inbred process.

\section{Runs of homozygosity}

Runs of homozygosity ( $\mathrm{ROH}$ ) are regions of the genome where the identical haplotypes are inherited from each parent. The exploration of $\mathrm{ROH}$ can help to identify recessive disease variants and investigate the effects of genome-wide homozygosity on traits of biomedical importance. To investigate homozygosity distribution in six RCS lines as well as the parental line $6_{3}$ and line $7_{2}$, we performed $\mathrm{ROH}$ analysis with PLINK using a total of 527,021 SNPs [17]. A sliding window of 50 SNPs was used to identify $\mathrm{ROH}$ based on filtered SNPs. Here, homozygous segments were defined as more than 50 homozygous SNPs spanning larger than $500 \mathrm{~kb}$. To avoid underestimation of $\mathrm{ROHs}$ caused by occasional genotyping error or missing genotype occurring in an otherwise-unbroken homozygous segment, we used the option --homozyg-window-het 1 --homozyg-window-missing 2 in PLINK to allow one heterozygous and two missing calls per window.

\section{Gene Ontology (GO) enrichment analysis}

To assess the function of the positive selected genes identified in top $1 \%$ windows regions and genes in the $\mathrm{ROH}$ segments, we performed gene enrichment annotation and gene functional classification with DAVID (version 6.7) [23]. The default sets were used in DAVID with gene annotations based on Gallus gallus, and GOTERM_BP_FAT, GOTERM_CC_FAT, GOTERM_MF_FAT were selected as the functional annotation category.

\section{Results}

Summary statistics of SNPs array of QC

The quality of all SNPs in the array met the 99\% call rate (Additional file 1: Figure S2). In the present study, a total of 527,021 SNPs in autosome chromosomes were kept for further analysis. Markers with high missing gencall rate $(>0.1)$, low MAF $(<0.01)$ and significant deviation from Hardy-Weinberg equilibrium $\left(P<1 \times 10^{-6}\right)$ were excluded, leaving a total of 155,216 autosomal SNPs in population genetic structure analysis. Distributions in autosomes for total SNPs and filtered SNPs were presented in Additional file 1: Figures S3 and S4.

\section{Genetic structure and phylogenetic analysis}

MDS analysis revealed the first dimension (C1) and the second dimension $(\mathrm{C} 2)$ separated the lines into three broad non-overlapping clusters, which represent line $7_{2}$ (susceptibility), line $6_{3}$ (resistance), RCS lines and F1 (hybrid F1 generation with various level of resistance). Six F1 chickens clustered together, and they were distinguished from both susceptibility and resistance lines. We also observed 18 chickens from RCS lines were specifically clustered into resistance sub-groups distributed around line $6_{3}$ (Fig. 1a). Moreover, we further investigated genetic structure within 18 RCS chickens using MDS analysis, and observed J lines separated clearly from others, which indicated a differential genomic signature in J (Fig. 1b). Other lines also displayed obviously independent cluster on the MDS plots. Additionally, the results from heatmap and neighbor-joining (NJ) tree were consistent with MDS results (Fig. 2), we also found line $7_{2}$, next to F1 hybrid lines, were divergent from other lines.

Then, we employed a model-based unsupervised hierarchical clustering across chickens using the program STRUCTURE. The admixture bar plot results were in well agreement with above observations with a clear separation of line $7_{2}, \mathrm{~F} 1$, line $6_{3}$ and others, as shown in Fig. 3 (e.g. $\mathrm{K}=3$ ). In addition, as $\mathrm{K}$ increases to 4 , J line was separated from other lines. Interestingly, when $K=10$, we found line $C, J, N, X$ were separated from 

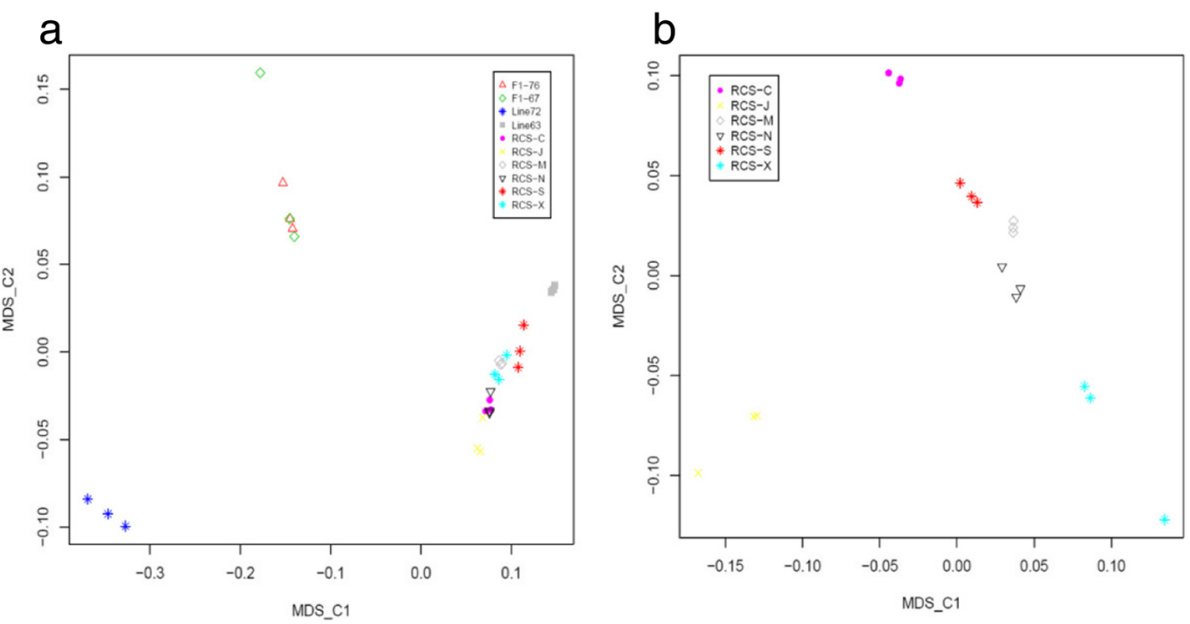

Fig. 1 a Multidimensional scaling analysis (MDS) based on genome-wide IBS pairwise distances in a total of 30 chickens. b Multidimensional scaling analysis based on genome-wide IBS pairwise distances within 18 RCS chickens

line $6_{3}$ (indicating unique resistance lines). In addition, the neighbor-joining $(\mathrm{NJ})$ tree was constructed based on IBD calculated between unambiguously separated individuals, and line $7_{2}$ was used as control group (Fig. 4). The results indicated detailed genetic relationships among 30 chickens as shown in Fig. 2. Three groups of inbreed lines corresponding to line $7_{2}$, line $6_{3}$, F1 could be clearly distinguished in upper, lower right and lower left, respectively. The results were also consistent with MDS and Admixture results: three individuals from different lines were clustered together at the same branches and individuals from F1 branched in an intermediary position between line $7_{2}$ and line $6_{3}$. Most importantly, all RCS lines were closely related to the line $6_{3}$ MD-resistant lines, which suggesting a higher influence from the genetics background of line

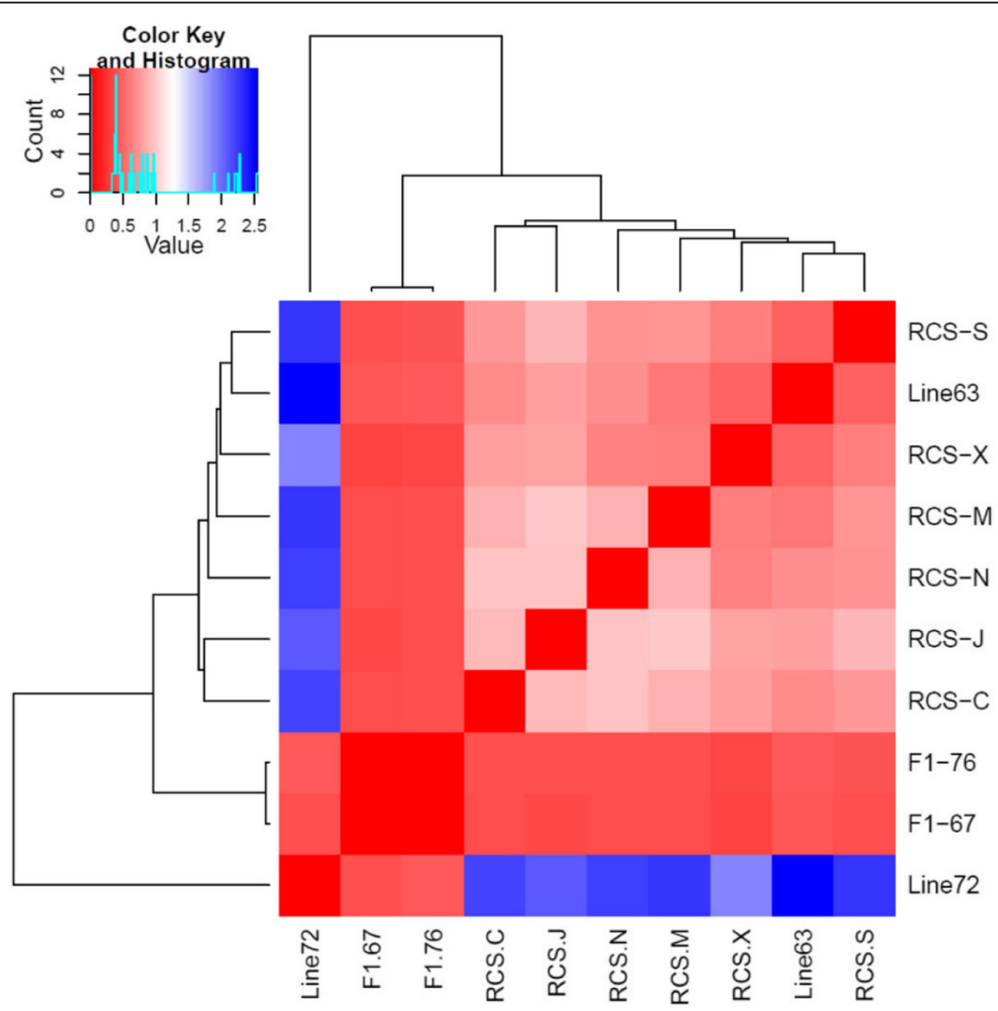

Fig. 2 Heatmap and hierarchical clustering tree based on the Reynolds' distance between 10 inbred lines 


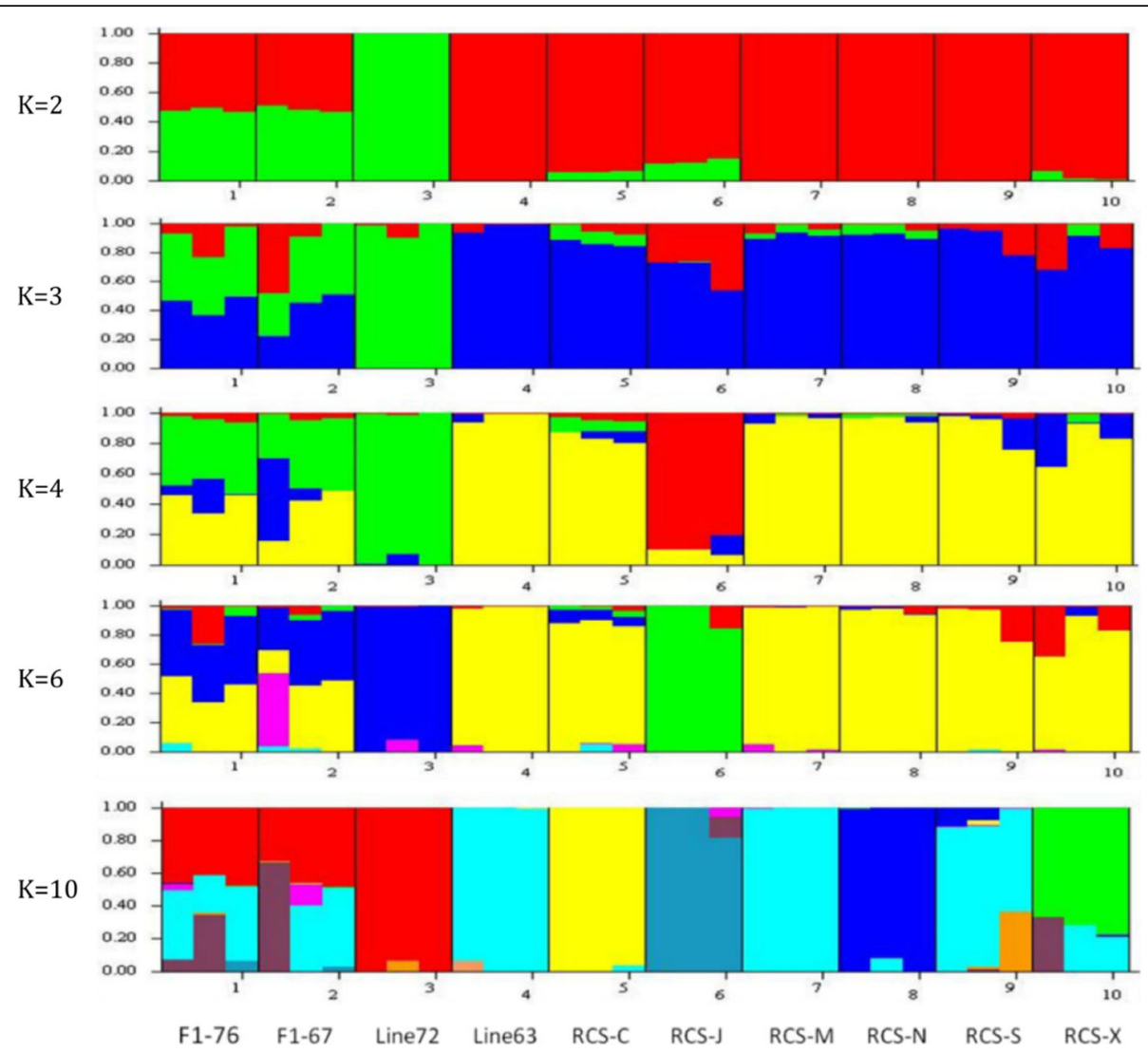

Fig. 3 Admixture analysis using LD filtered 5,064 SNPs in all 30 chickens. Chickens are indicated by thin vertical lines partitioned into segments corresponding to the inferred membership in $\mathrm{K}=2, \mathrm{~K}=3, \mathrm{~K}=4, \mathrm{~K}=6$ and $\mathrm{K}=10$ genetic clusters as indicated by the colors

6. In addition, we found chickens from line $\mathrm{X}, \mathrm{N}$ and chickens from $\mathrm{S}, \mathrm{M}, \mathrm{C}$, J were readily distinguishable among RCS lines, which suggested that these lines could have their unique genetic characteristics as previously reported [10].

\section{Genomic diverse signatures}

To explore the difference of genetic polymorphism related to susceptibility and resistance during the inbreeding process, we carried out genome scan across genome using $F_{\mathrm{ST}}$ approach. After splitting the whole genome to 50 SNP windows, we obtained 10,554 raw windows. We detected 106 regions (about top 1\%) with diverse signatures. To gain insight of the function enrichment of these regions, we annotated the top 106 candidate regions, and 51 genes were identified to be candidate genes for the resistance to MD. To better understand the biological processes and molecular function involved in MD genetic resistance, we performed GO term analysis using DAVID. We observed the most represented molecular function was arylamine $\mathrm{N}$-acetyltransferase activity (GOTERM_MF_FAT), which included gene PNAT10 (N-acetyltransferase, pineal gland isozyme NAT-10), PNAT3 (N-acetyltransferase, pineal gland isozyme NAT-3), and NAT (N-acetyltransferase, liver isozyme) (Table 1). The most represented biological process was regulation of cell death and anti-apoptosis (GOTERM_BP_FAT). Those genes included AKT1 (v-akt murine thymoma viral oncogene homolog 1 ), CDH13 (cadherin 13, H-cadherin), CFDP1 (craniofacial development protein 1), API5 (apoptosis inhibitor 5), and ING4 (inhibitor of growth family, member 4). Furthermore, KEGG_PATHWAY analysis identified PNAT10, PNAT3, NAT involved pathway caffeine metabolism and drug metabolism (Additional file 2: Table S1). We also detected many overlaps between the candidate regions with online chicken QTL database (http:// www.animalgenome.org/cgi-bin/QTLdb/GG/index). The overlapping positions of each region and QTLs related to MD disease were listed in Additional file 3: Table S2. We observed 19 regions overlapping with known QTL regions, including 9 regions in chr1 [7, 8], 2 regions at chr2 $[8,24], 5$ regions in chr5 and 1 region in chr9 $[7,8]$.

\section{Runs of homozygosity}

In this study, we found many loci had been fixed to homozygous. It is impossible to use them to explain the difference of MD resistance. However, the completed differentiations 


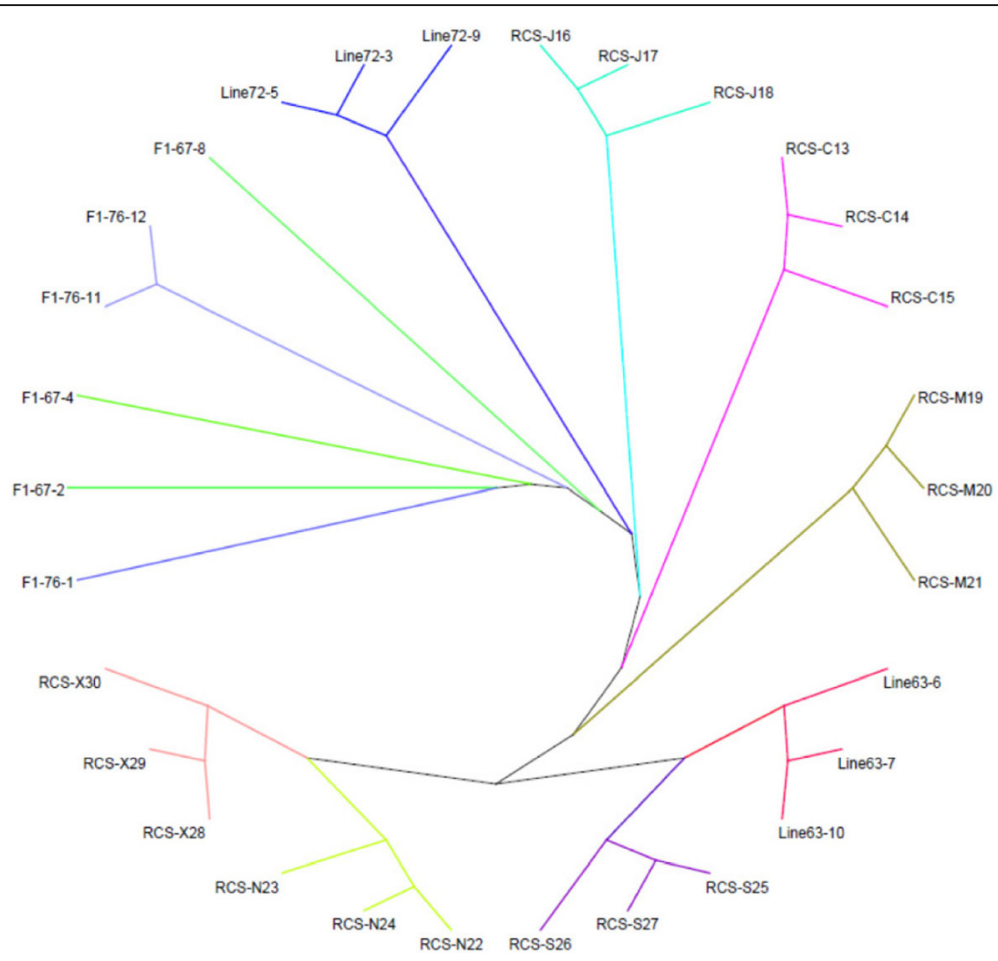

Fig. 4 Neighbor-joining tree based on genome-wide IBD distances in 30 chickens

$\left(F_{\mathrm{ST}}=1\right)$ indicates two different homozygous genotypes existed in populations. To estimate the inbred level across lines, we also calculated the inbreeding coefficient $(F)$ for all 30 samples using 155,216 high quality SNPs (Additional file 4: Table S3). We found the top inbreeding coefficient $(F)$ in line $6_{3}$, which is over 0.98 . Next is line $7_{2}$, nearly 0.95 . In contrast, the average of $F$ among the RCS is $0.88 \pm 0.028$. Among them, the average of $F$ values in RCS-S line is up to 0.94 , whereas for lines $\mathrm{N}$ and $\mathrm{X}, F$ values are both about 0.86, which suggested there existed slight different inbred levels among inbred chicken lines.
To explore the question whether the inbred levels and homozygosity differences across the genome could contribute to the susceptibility/resistance for MD, we conducted homozygosity analysis across all 24 chickens (hybrid F1 lines were not included in $\mathrm{ROH}$ analysis). We obtained 2,027 ROH segments, and 1,596 of them were found in RCS lines. The genome wide results from the $\mathrm{ROH}$ analysis indicated obviously different $\mathrm{ROH}$ distributions across the genome within 24 inbred chickens. We identified $406 \mathrm{ROH}$ segments in line $7_{2}$ and $206 \mathrm{ROH}$ segments in line $6_{3}$. We also found $\mathrm{ROH}$

Table 1 Gene Ontology annotations of candidate genes identified using F FT approach

\begin{tabular}{lllll}
\hline GO category & GO term & Gene count & P-value & List of genes \\
\hline GOTERM_BP_FAT & GO:0006916 anti-apoptosis & 4 & $2.40 \mathrm{E}-04$ & AKT1, CDH13, CFDP1, API5 \\
GOTERM_BP_FAT & GO:0042981 regulation of apoptosis & 5 & $2.70 \mathrm{E}-03$ & AKT1, CDH13, ING4, CFDP1, API5 \\
GOTERM_BP_FAT & GO:0043067 regulation of programmed cell death & 5 & $2.89 \mathrm{E}-03$ & AKT1, CDH13, ING4, CFDP1, API5 \\
GOTERM_BP_FAT & GO:0010941 regulation of cell death & 5 & $2.94 \mathrm{E}-03$ & AKT1, CDH13, ING4, CFDP1, API5 \\
GOTERM_BP_FAT & GO:0043066 negative regulation of apoptosis & 4 & $3.26 \mathrm{E}-03$ & AKT1, CDH13, CFDP1, API5 \\
GOTERM_BP_FAT & GO:0043069 negative regulation of programmed cell death & 4 & $3.44 \mathrm{E}-03$ & AKT1, CDH13, CFDP1, API5 \\
GOTERM_BP_FAT & GO:0060548 negative regulation of cell death & 4 & $3.44 \mathrm{E}-03$ & AKT1, CDH13, CFDP1, API5 \\
GOTERM_MF_FAT & GO:0004060 arylamine N-acetyltransferase activity & 3 & $5.20 \mathrm{E}-05$ & PNAT10, PNAT3, NAT \\
GOTERM_MF_FAT & GO:0008080 N-acetyltransferase activity & 3 & $2.12 \mathrm{E}-03$ & PNAT10, PNAT3, NAT \\
GOTERM_MF_FAT & GO:0016410 N-acyltransferase activity & 3 & $2.71 \mathrm{E}-03$ & PNAT10, PNAT3, NAT \\
GOTERM_MF_FAT & GO:0016407 acetyltransferase activity & 3 & $3.14 \mathrm{E}-03$ & PNAT10, PNAT3, NAT
\end{tabular}


distribution displayed a certain degree of diversity among the six RCS lines, with the longest $\mathrm{ROH}$ detected in line $6_{3}$ and line $\mathrm{N}$, about $90 \mathrm{Mb}$. However, in line $7_{2}$, the longest $\mathrm{ROH}$ was $44 \mathrm{Mb}$. The detailed summary statistics for $\mathrm{ROH}$ analysis, including $\mathrm{ROH}$ number, length and density (nSNP/kb) and SNPs within each $\mathrm{ROH}$ segment, was shown in Additional file 5: Table S4.

To compare the $\mathrm{ROH}$ distributions among 8 diverse inbred lines, we first merged $\mathrm{ROH}$ segments identified from all individuals for each line into nonredundent $\mathrm{ROH}$ regions based on genomics coordinate. We finally obtained 58, 71, 59, 64, 65, 63, 58 and $67 \mathrm{ROH}$ regions in the lines $6_{3}, 7_{2}, C, J, M, N, S$ and $X$, respectively, while the relative more $\mathrm{ROH}$ regions were identified on chr1 (10 ROHs) in line 72 and chr2 (8 ROHs) in line L. The most probable reason was both chromosomes cover a larger proportion of genome size as compared with other chromosomes. To facilitate the comparison of difference of $\mathrm{ROH}$ regions among lines, genome wide $\mathrm{ROH}$ regions were presented in Additional file 5: Table S4. We found some shared $\mathrm{ROH}$ regions, both among the RCS lines and between RCS and line $6_{3}$. The results indicated that $\mathrm{ROH}$ region caused by inbreeding may be involved in the different MD resistance. On the other hand, the unique region from line $7_{2}$ (susceptibility line) comparing other lines may offer some clues of genomic homozygosity region related to susceptibility.

$\mathrm{ROH}$ represents regions of genome where the identical haplotype is inherited from each parent. $\mathrm{ROH}$ regions could likely increase of recessive deleterious alleles to be co-contributed, and reducing the viability of the organism. To investigate the differential $\mathrm{ROH}$ distribution among diverse chickens, we further divided 24 chickens into three groups, representing parental line $6_{3}$ with resistance, parental susceptibility line $7_{2}$ group, and RCS group (including line $\mathrm{C}, \mathrm{J}, \mathrm{M}, \mathrm{N}, \mathrm{S}$ and $\mathrm{X}$ ), respectively. Comparisons of $\mathrm{ROH}$ regions based on genomics coordinates among three groups showed that 98.6\% ROH region $(902.5 / 915.0 \mathrm{Mb})$ were shared by all three groups (Additional file 1: Figure S5).

In our study, two parental inbred line $6_{3}$ and line $7_{2}$ are MD-resistant and MD-susceptible respectively, while a Recombinant Congenic Strains (RCS) lines were developed from these two lines showing varying resistance to MD, we observed RCS lines were similar to line 63 based on genetics relation estimation in present study (Figs. 1, 2, 3 and 4). To further explore whether homozygous segments segregated with different resistance to MD diseases, all chickens with varying resistance to MD included RCS lines and line $6_{3}$ chickens were compared with the line $7_{2}$ (MD-susceptible). We obtained one region that is uniquely distributed in line $6_{3}$ with $513 \mathrm{~kb}$ length; eight regions (987 kb in size) in line $7_{2}$ groups, and 47 regions totaling $7,719 \mathrm{~kb}$ not in line 72 but in shared by both RCS lines and line $6_{3}$ (Additional file 6: Table S5).

Subsequently, we observed 56 genes in 47 shared $\mathrm{ROH}$ regions, while only one gene was identified within line-specific regions in the line $6_{3}$. We identified eight unique regions with 13 genes in line $7_{2}$. Among the 56 genes identified in both line $6_{3}$ and RCS with different resistance, we found most of them were enriched in regulation of apoptosis and regulation of cell death, this result may indicate their potential association with the resistance of MD disease.

\section{Discussion}

We investigated the genetic characteristics of MD resistance and susceptibility using high-density SNP array in inbred chicken lines. Genetic structure indicated differential genomic landscapes during inbreeding process in the past decades. Using genomic signature and homozygosity analysis, we identified a list of genes that may be contribute to the MD resistance or susceptibility in the inbred lines.

Previous studies have explored the genetic changes at gene expression level [10], epigenetic regulatory $[25,26]$, and allele specific expression [11] in the inbred chickens. To better understand the genetic mechanism underlying MD-resistance and susceptibility, we employed high density SNP arrays to investigate the genetic structure and relationship in 10 inbred lines. Using MDS analysis, we observed diverse genetic structures among them, which were highly consistent with admixture analysis and phylogenetic analysis. These chicken lines with different MD susceptibilities were separated obviously, while RCS lines were clustered together near parental line $6_{3}$ with similar MD-resistance. Our findings were also consistent with PCA analysis in inbred chicken using expression array [10], these results indicated similar influences on MD-resistance and susceptibility caused by SNPs and gene expression.

Although the limited samples size per line were utilized in the current study, the global $\mathrm{F}_{S T}$ approach can properly reflect the potential difference among all 30 birds. Allele frequency based method is likely to offer some important clues for searching the candidate genes involved in the MD resistance. Moreover, we found some genes related to disease and immune response. For instance, several genes were significant enriched including arylamine $\mathrm{N}$-acetyltransferase activity (AKT1, CDH13, CFDP1, API5) and cell death and anti-apoptosis (CDH13, ING4, CFDP1 and API5). We also detected one region located at $51.5 \mathrm{Mb}$ in chromosome 5 with high selection signal $\left(F_{\mathrm{ST}}=0.574986\right)$ that contained gene AKT1. AKT1 (protein kinase $\mathrm{B}, \mathrm{PKB}$ ) is a serine/ threonine kinase that plays a critical role in regulating cell 
survival, insulin signaling, angiogenesis and tumor formation [27-29]. Previous studies in chicken have revealed AKT1 as one of candidate gene for Salmonella response [30]. Moreover, subsequent fine-mapping of heterophil functional response to Salmonella in a highly advanced intercross revealed the position containing both $A K T 1$ and SIVA may work as heterophil function to explain the host-resistance properties [31]. Another gene identified in our analysis was $\mathrm{CDH} 13$ was located at $15.6 \mathrm{Mb}$ in chromosome 11. This gene encodes a member of the cadherin superfamily, which is hypermethylated in many types of cancer. Previous studies have showed that CDH13 may be a promising candidate gene for Attention Deficit/Hyperactivity Disorder $(A D H D)[32,33]$ and plays a central role in the regulation of brain networks [34]. Intriguingly, we found an apoptosis inhibitory protein, coded by gene API5 located at $20 \mathrm{Mb}$ in chromosome. This apoptosis inhibitory protein prevents apoptosis after growth factor deprivation and suppresses the transcription factor E2F1-induced apoptosis, and negatively regulating acinus, a nuclearfactor involved in apoptotic DNA fragmentation. Its depletion enhances the cytotoxic action of the chemotherapeutic drugs [35].

$\mathrm{ROH}$ regions can increase the recessive deleterious alleles to be co-contributed, and reduce the viability of the organism. Differential $\mathrm{ROH}$ segments across lines may contain potential candidate genes related to $M D$ resistance. In this study, 5 genes ( $S I K, S O X 1$, LIG4, SIK1 and TNFSF13B) within $\mathrm{ROH}$ region were found in resistant group (line $6_{3}$ and RCS), and these genes have been previously reported that may be related to immunology and survival. Previous studies demonstrated that SIK1 gene plays critical role in promoting survival of skeletal myocytes using mouse model [36] and involve with regulation of its abundance and stability for myogenesis [37]. Sox 1 as a part of the $S o x-B 1$ group of transcriptional regulators in neural progenitor cells is sufficient to induce neuronal lineage commitment [38]. Also, it promotes neuronal cell fate determination and differentiation by integrating multiple independent pathways. In human, the GG genotype of LIG4 was found to be associated with higher IgE levels to Ascaris [39]. Also, gene TNFSF13B, as one of BAFF receptors, was proved to be a key survival factor during B-cell maturation [40, 41].

Interestingly, we identified one unique $\mathrm{ROH}$ region (at $110 \mathrm{Mb}$ in chromosome 2) in line $7_{2}$, which contains five genes LYN, MOS, PLAG1, TGS1 and TMEM68. Among them, $L Y N$ regulates survival and responsiveness of tumor cells by a BCR-ABL1 independent mechanism. In various hematopoietic cells, LYN has emerged as a key enzyme involved in the regulation of cell activation. In these cells, a small amount of LYN is associated with cell surface receptor proteins, including the B cell antigen receptor (BCR) [42], and CD40 [43]. PLAG1 (pleiomorphic adenoma gene 1) was found frequently rearranged and activated in human salivary gland pleomorphic adenomas. It encodes a developmentally regulated transcription factor [44]. Ectopic overexpression of PLAG1 has been proposed to play a crucial role in tumorigenesis of salivary gland pleomorphic adenomas. It was reported that PLAG1 can activate the transcription of insulin-like growth factor 2 (IGF2), functioning as a proto-oncogene [45]. PLAG1 is a proto-oncogene whose overexpression is a crucial oncogenic event in salivary gland pleomorphic adenomas (PA), and in carcinoma ex pleomorphic adenoma (CA-ex-PA). Our results provided some important insights for understanding the molecular mechanisms of MD resistance. Moreover, we identified gene USP15 (ubiquitin-specific protease 15) in line $6_{3}$, which may indicate its potential role for MD resistance. USP15 have been reported that have an essential role for regulation of caspase-3 during Paclitaxel-induced apoptosis [46] and TGF- $\beta$ pathway in human studies [47].

\section{Conclusions}

We investigated the genomic characteristics of inbred chicken lines using high density SNP array by integrating genomic signature, $\mathrm{ROH}$ analysis. Our findings revealed that several candidate genes including $A K T 1, C D H 13$, CFDP, API5, and USP15 for MD susceptibility. Future studies with large sample size and fine mapping of the genetic variants using powerful association statistics would elucidate the complex genetic mechanisms of resistance/susceptibility to $\mathrm{MD}$.

\section{Additional files}

Additional file 1: Figure S1. Histogram plot of MD incidence (\%) rate. MD resistance in chickens is generally evaluated with MD incidence (induced gross tumors by MDV) and survival day post MDV challenge, and the extent of resistance is dependent of the virulence of challenge viruses and other factors. Figure S2. Barplot show the call rate for 30 chickens on Affymetrix chicken SNP genotyping array. $X$ axis represent the call rate values, and $Y$ axis represents the chicken individuals. Figure S3. Frequency distribution in autosomes for a total of 527,021 SNPs on the Affymetrix chicken SNP genotyping array. Figure S4. Frequency distribution in autosomes for 155,216 SNPs on the Affymetrix chicken SNP genotyping array. Figure S5. Comparison of $\mathrm{ROH}$ regions among three groups, line $\sigma_{3}$, line $7_{2}$, lines $\mathrm{RCS}(\mathrm{C}$, $L, M, N, S, X)$. The overlap length of $\mathrm{ROH}$ was indicated in $\mathrm{kb}$. (DOCX $35 \mathrm{~kb}$ )

Additional file 2: Table S1. GO analysis for 51 genes in 106 identified candidate regions. (XLSX $20 \mathrm{~kb}$ )

Additional file 3: Table S2. The overlapping positions for 106 identified candidate regions with QTLs related to MD disease. (XLSX $12 \mathrm{~kb}$ )

Additional file 4: Table S3. The inbreeding coefficient $(F)$ for all 30 samples using 155,216 high quality SNPs. (XLSX $11 \mathrm{~kb}$ )

Additional file 5: Table S4. Summary statistics and $\mathrm{ROH}$ distributions among 8 diverse inbred lines including lines $\sigma_{3}, 7_{2}, C, J, M, N, S$ and $X$. (XLSX $31 \mathrm{~kb})$ 
Additional file 6: Table S5. Unique and shared homozygous segments across all chickens included RCS lines and line $\sigma_{3}$ chickens were compared with the line 72. (XLSX $13 \mathrm{~kb}$ )

\section{Abbreviations}

ADOL: Avian disease and oncology laboratory; BC: Reciprocal backcross; IBD: Identity-by-descent; IBS: Identical by state; LD: Linkage disequilibrium; MD: Marek's disease; MDS: Multidimensional scaling; MDV: Marek's disease virus; NJ: Neighbor-joining; QTL: Quantitative trait loci; RCS: Recombinant congenic strains; $\mathrm{ROH}$ : Runs of homozygosity

\section{Acknowledgements}

We thank Reuben Anderson and Alexandre Dimitriv for technical assistance.

\section{Funding}

The work was supported in part by U.S. Department of Agriculture (USDA)Nation Research Initiative/National Institute of Food and Agriculture (NRI/NIFA) 2008-35204-04660, 2010-65205-20588 and 2011-67015-30183. Mention of trade names or commercial products in this article is solely for the purpose of providing specific information and does not imply recommendation or endorsement by USDA. USDA is an equal opportunity provider and employer.

\section{Availability of data and materials}

The Genotype data reported in this article are available upon request for research.

\section{Authors' contributions}

JZS and LYX conceived and designed the experiments. LYX, YHH and YD performed data analyses. HHC, HMZ, and RLT collected samples and generated the SNP genotyping data. LYX, GEL and JZS wrote the paper. All authors read and approved the final manuscript.

\section{Ethics approval and consent to participate}

All of the chickens were kept in a pathogen-free facility at the Avian Disease and Oncology Laboratory (ADOL). All animals were approved by the United States Department of Agriculture, Agricultural Research Service, ADOL Animal Care and Use Committee and followed their guidelines (revised April 2005) as well as the Guide for the Care and Use of Laboratory Animals published by Institute for Laboratory Animal Research (ILAR Guide) in 1996 (http://www.nap.edu/openbook.php?record id=5140) and University of Maryland (R-08-62)

\section{Consent for publication}

Not applicable.

\section{Competing interests}

The authors declare that they have no competing interests.

\section{Author details}

'Department of Animal \& Avian Sciences, University of Maryland, College Park, MD 20742, USA. ${ }^{2}$ Animal Genomics and Improvement Laboratory, U.S. Department of Agriculture-Agricultural Research Services, Beltsville, MD 20705, USA. ${ }^{3}$ Laboratory of Molecular Biology and Bovine Breeding, Institute of Animal Science, Chinese Academy of Agricultural Sciences, Beijing 100193, China. ${ }^{4}$ United States Department of Agriculture-Agriculture Research Service (ARS), Avian Disease and Oncology Laboratory, East Lansing, MI 48823, USA. ${ }^{5}$ Animal and Nutritional Sciences, Davis College of Agriculture, West Virginia University, Morgantown, WV 26506, USA.

Received: 7 April 2018 Accepted: 25 July 2018

Published online: 13 September 2018

\section{References}

1. Lee LF, Powell PC, Rennie M, Ross LJ, Payne LN. Nature of genetic resistance to Marek's disease in chickens. J Natl Cancer Inst. 1981;66:789-96.

2. Bacon LD, Hunt HD, Cheng HH. A review of the development of chicken lines to resolve genes determining resistance to diseases. Poult Sci. 2000;79: 1082-93.

3. Chang S, Dunn JR, Heidari M, Lee LF, Song J, Ernst CW, et al. Genetics and vaccine efficacy: host genetic variation affecting Marek's disease vaccine efficacy in White Leghorn chickens. Poult Sci. 2010;89:2083-91.
4. Osterrieder N, Kamil JP, Schumacher D, Tischer BK, Trapp S. Marek's disease virus: from miasma to model. Nat Rev Microbiol. 2006:4:283-94.

5. Burgess SC, Young JR, Baaten BJ, Hunt L, Ross LN, Parcells MS, et al. Marek's disease is a natural model for lymphomas overexpressing Hodgkin's disease antigen (CD30). Proc Natl Acad Sci U S A. 2004;101:13879-84.

6. Cheng HH, Kaiser P, Lamont SJ. Integrated genomic approaches to enhance genetic resistance in chickens. Annu Rev Anim Biosci. 2013;1:239-60.

7. Heifetz EM, Fulton JE, O'Sullivan NP, Arthur JA, Wang J, Dekkers JC, Soller M. Mapping quantitative trait loci affecting susceptibility to Marek's disease virus in a backcross population of layer chickens. Genetics. 2007;177:2417-31.

8. Heifetz EM, Fulton JE, O'Sullivan NP, Arthur JA, Cheng H, Wang J, et al. Mapping QTL affecting resistance to Marek's disease in an F6 advanced intercross population of commercial layer chickens. BMC Genomics. 2009;10:20.

9. Liu HC, Kung HJ, Fulton JE, Morgan RW, Cheng HH. Growth hormone interacts with the Marek's disease virus SORF2 protein and is associated with disease resistance in chicken. Proc Natl Acad Sci U S A. 2001;98:9203-8.

10. Yu Y, Luo J, Mitra A, Chang S, Tian F, Zhang H, et al. Temporal transcriptome changes induced by MDV in Marek's disease-resistant and -susceptible inbred chickens. BMC Genomics. 2011;12:501.

11. Perumbakkam S, Muir WM, Black-Pyrkosz A, Okimoto R, Cheng HH. Comparison and contrast of genes and biological pathways responding to Marek's disease virus infection using allele-specific expression and differential expression in broiler and layer chickens. BMC Genomics. 2013;14:64.

12. Xu L, He Y, Ding Y, Sun G, Carrillo JA, Li Y, et al. Characterization of copy number variation's potential role in Marek's disease. Int J Mol Sci. 2017;18:1020.

13. Chang S, Ding Z, Dunn JR, Lee LF, Heidari M, Song J, et al. A comparative evaluation of the protective efficacy of rMd5deltaMeq and CVI988/ Rispens against a v + strain of Marek's disease virus infection in a series of recombinant congenic strains of White Leghorn chickens. Avian Dis. 2011;55:384-90.

14. Luo J, Yu Y, Mitra A, Chang S, Zhang H, Liu G, et al. Genome-wide copy number variant analysis in inbred chickens lines with different susceptibility to Marek's disease. G3. 2013:3:217-23.

15. Pritchard JK, Stephens M, Donnelly P. Inference of population structure using multilocus genotype data. Genetics. 2000;155:945-59.

16. Falush D, Stephens M, Pritchard JK. Inference of population structure using multilocus genotype data: linked loci and correlated allele frequencies. Genetics. 2003;164:1567-87.

17. Purcell S, Neale B, Todd-Brown K, Thomas L, Ferreira MA, Bender D, et al. PLINK: a tool set for whole-genome association and population-based linkage analyses. Am J Hum Genet. 2007;81:559-75.

18. Stevens EL, Heckenberg G, Roberson ED, Baugher JD, Downey TJ, Pevsner J. Inference of relationships in population data using identity-by-descent and identity-by-state. PLoS Genet. 2011;7:e1002287.

19. Excoffier $L$, Lischer HE. Arlequin suite ver 3.5: a new series of programs to perform population genetics analyses under Linux and Windows. Mol Ecol Resour. 2010;10:564-7

20. Rousset F. genepop'007: a complete re-implementation of the genepop software for Windows and Linux. Mol Ecol Resour. 2008:8:103-6.

21. Weir BS, Hill WG. Estimating F-statistics. Annu Rev Genet. 2002;36:721-50.

22. Akey JM, Zhang G, Zhang K, Jin L, Shriver MD. Interrogating a high-density SNP map for signatures of natural selection. Genome Res. 2002;12:1805-14.

23. Huang DW, Sherman BT, Tan Q, Kir J, Liu D, Bryant D, et al. DAVID Bioinformatics Resources: expanded annotation database and nove algorithms to better extract biology from large gene lists. Nucleic Acids Res. 2007;35:W169-75.

24. Yonash N, Bacon LD, Witter RL, Cheng HH. High resolution mapping and identification of new quantitative trait loci (QTL) affecting susceptibility to Marek's disease. Anim Genet. 1999;30:126-35.

25. Mitra A, Luo J, Zhang H, Cui K, Zhao K, Song J. Marek's disease virus infection induces widespread differential chromatin marks in inbred chicken lines. BMC Genomics. 2012;13:557.

26. Luo J, Mitra A, Tian F, Chang S, Zhang H, Cui K, et al. Histone methylation analysis and pathway predictions in chickens after MDV infection. PLoS One 2012:7:e41849.

27. Datta SR, Brunet A, Greenberg ME. Cellular survival: a play in three Akts. Genes Dev. 1999:13:2905-27.

28. Carpten JD, Faber AL, Horn C, Donoho GP, Briggs SL, Robbins CM, et al. A transforming mutation in the pleckstrin homology domain of AKT1 in cancer. Nature. 2007;448:439-44.

29. Brugge J, Hung MC, Mills GB. A new mutational AKTivation in the PI3K pathway. Cancer Cell. 2007;12:104-7. 
30. Fife MS, Salmon N, Hocking PM, Kaiser P. Fine mapping of the chicken salmonellosis resistance locus (SAL1). Anim Genet. 2009;40:871-7.

31. Redmond SB, Chuammitri P, Andreasen CB, Palic D, Lamont SJ. Genetic control of chicken heterophil function in advanced intercross lines: associations with novel and with known Salmonella resistance loci and a likely mechanism for cell death in extracellular trap production. Immunogenetics. 2011;63:449-58.

32. Franke B, Faraone SV, Asherson P, Buitelaar J, Bau CH, Ramos-Quiroga JA, et al. AC International Multicentre persistent. The genetics of attention deficit/ hyperactivity disorder in adults, a review. Mol Psychiatry. 2012;17:960-87.

33. Jee SH, Sull JW, Lee JE, Shin C, Park J, Kimm H, et al. Adiponectin concentrations: a genome-wide association study. Am J Hum Genet. 2010; 87:545-52.

34. Rivero O, Sich S, Popp S, Schmitt A, Franke B, Lesch KP. Impact of the ADHD-susceptibility gene $\mathrm{CDH} 13$ on development and function of brain networks. Eur Neuropsychopharmacol. 2013;23:492-507.

35. Morris EJ, Michaud WA, Ji JY, Moon NS, Rocco JW, Dyson NJ. Functional identification of Api5 as a suppressor of E2F-dependent apoptosis in vivo. PLoS Genet. 2006;2:e196.

36. Berdeaux R, Goebel N, Banaszynski L, Takemori H, Wandless T, Shelton GD, Montminy M. SIK1 is a class II HDAC kinase that promotes survival of skeletal myocytes. Nat Med. 2007:13:597-603.

37. Stewart R, Akhmedov D, Robb C, Leiter C, Berdeaux R. Regulation of SIK1 abundance and stability is critical for myogenesis. Proc Natl Acad Sci U S A. 2013;110:117-22.

38. Kan L, Israsena N, Zhang Z, Hu M, Zhao LR, Jalali A, et al. Sox1 acts through multiple independent pathways to promote neurogenesis. Dev Biol. 2004; 269:580-94.

39. Acevedo N, Mercado D, Vergara C, Sanchez J, Kennedy MW, Jimenez S, et al. Association between total immunoglobulin $\mathrm{E}$ and antibody responses to naturally acquired Ascaris lumbricoides infection and polymorphisms of immune system-related LIG4, TNFSF13B and IRS2 genes. Clin Exp Immunol. 2009;157:282-90.

40. Mackay F, Silveira PA, Brink R. B cells and the BAFF/APRIL axis: fast-forward on autoimmunity and signaling. Curr Opin Immunol. 2007;19:327-36.

41. Mackay F, Schneider P, Rennert P, Browning J. BAFF AND APRIL: a tutorial on B cell survival. Annu Rev Immunol. 2003:21:231-64.

42. Mlinaric-Rascan I, Yamamoto T. B cell receptor signaling involves physical and functional association of FAK with Lyn and IgM. FEBS Lett. 2001:498:26-31.

43. Ren CL, Morio T, Fu SM, Geha RS. Signal transduction via CD40 involves activation of lyn kinase and phosphatidylinositol-3-kinase, and phosphorylation of phospholipase C gamma 2. J Exp Med. 1994;179:673-80.

44. Van Dyck F, Declercq J, Braem CV, Van de Ven WJ. PLAG1, the prototype of the PLAG gene family: versatility in tumour development (review). Int J Oncol. 2007;30:765-74.

45. Zhao $X$, Ren $W$, Yang $W$, Wang $Y$, Kong $H$, Wang L, et al. Wnt pathway is involved in pleomorphic adenomas induced by overexpression of PLAG1 in transgenic mice. Int J Cancer. 2006;1 18:643-8.

46. Xu M, Takanashi M, Oikawa K, Tanaka M, Nishi H, Isaka K, et al. USP15 plays an essential role for caspase-3 activation during Paclitaxel-induced apoptosis. Biochem Biophys Res Commun. 2009;388:366-71.

47. Eichhorn PJ, Rodon L, Gonzalez-Junca A, Dirac A, Gili M, Martinez-Saez E, et al. USP15 stabilizes TGF-beta receptor I and promotes oncogenesis through the activation of TGF-beta signaling in glioblastoma. Nat Med. 2012;18:429-35.

Ready to submit your research? Choose BMC and benefit from:

- fast, convenient online submission

- thorough peer review by experienced researchers in your field

- rapid publication on acceptance

- support for research data, including large and complex data types

- gold Open Access which fosters wider collaboration and increased citations

- maximum visibility for your research: over $100 \mathrm{M}$ website views per year

At $\mathrm{BMC}$, research is always in progress.

Learn more biomedcentral.com/submissions 